\title{
Impact of changing from staining to culture techniques on detection rates of Campylobacter spp. in routine stool samples in Chile
}

\author{
Lorena Porte*, Carmen Varela, Thomas Haecker, Sara Morales and Thomas Weitzel
}

\begin{abstract}
Background: Campylobacter is a leading cause of bacterial gastroenteritis, but sensitive diagnostic methods such as culture are expensive and often not available in resource limited settings. Therefore, direct staining techniques have been developed as a practical and economical alternative. We analyzed the impact of replacing Campylobacter staining with culture for routine stool examinations in a private hospital in Chile.

Methods: From January to April 2014, a total of 750 consecutive stool samples were examined in parallel by Hucker stain and Campylobacter culture. Isolation rates of Campylobacter were determined and the performance of staining was evaluated against culture as the gold standard. Besides, isolation rates of Campylobacter and other enteric pathogens were compared to those of past years.

Results: Campylobacter was isolated by culture in 46 of 750 (6.1 \%) stool samples. Direct staining only identified three samples as Campylobacter positive and reached sensitivity and specificity values of 6.5 and $100 \%$, respectively. In comparison to staining-based detection rates of previous years, we observed a significant increase of Campylobacter cases in our patients.

Conclusion: Direct staining technique for Campylobacter had a very low sensitivity compared to culture. Staining methods might lead to a high rate of false negative results and an underestimation of the importance of campylobacteriosis. With the inclusion of Campylobacter culture, this pathogen became a leading cause of intestinal infection in our patient population.
\end{abstract}

Keywords: Campylobacter, Diagnosis, Culture media, Staining methods

\section{Background}

The genus Campylobacter comprises fastidious S-shaped or spiral gram-negative bacteria with a length of 0.5 to $5 \mu \mathrm{m}$, which are microaerophilic, non-spore-forming, and mobile by the presence of a polar flagellum. Campylobacter is among the most important causes of foodborne infections causing human gastroenteritis worldwide [1]. Infections with this pathogen might be followed by severe sequelae such as reactive arthritis, Guillain-Barré syndrome, and irritable bowel syndrome [2]. Campylobacter species are ubiquitous in the environment and the intestinal tracts

\footnotetext{
* Correspondence: nenapor@gmail.com

Laboratorio Clínico, Clínica Alemana de Santiago, Facultad de Medicina

Clínica Alemana, Universidad del Desarrollo, Av. Vitacura 5951, Santiago, Chile
}

of a variety of free-living birds, wild and domestic food animals, and pets $[3,4]$. As an emerging zoonotic agent, it is of growing public health importance and also affects non-clinical areas such as food-production and animal handling [1].

There has been a rise in the incidence of campylobacteriosis in the last ten years in most developed regions such as North America, Europe, and Australia [5]. In South America, the role of Campylobacter as an enteric pathogen is less clear, since epidemiological data are scant and inconclusive $[1,5,6]$. In Chile, Campylobacter is a notifiable enteric pathogen. Recently, the National Reference Laboratory (Instituto de Salud Pública, Santiago, Chile) reported an average of 91 annual Campylobacter cases for 
the whole of Chile during 2005 to 2012, implicating incidence rates of 0.1 to $0.6 / 100.000$ [7]. In comparison, the current rate in the USA is $13.5 / 100.000$ [8]. A recent study from southern Chile detected C. jejuni in 10 and $10.7 \%$ of diarrheic samples by culture and PCR, respectively [9]. Older reports from the 1980s and 1990s, which mainly focused on pediatric populations, showed incidence rates of 10 and $16 \%$ in symptomatic children [10,11].

Culture methods are the gold standard for the diagnosis of intestinal Campylobacter infections. However, these methods require special selective media incubated at $37^{\circ}$ and/or $42{ }^{\circ} \mathrm{C}$ under microaerophilic conditions and are therefore inconvenient and expensive [12]. As a consequence, they are not included within the routine stool workup of many non-industrialized countries including Chile [7]. As a more economical alternative, direct stool staining methods have been developed and currently, these techniques are recommended in Chile for the routine detection of Campylobacter in stool samples of patients with acute gastroenteritis [13]. However, these methods are insufficiently evaluated and have drawbacks such as operator dependency. Due to sensitivity problems [14], they might underestimate the true incidence of this organism.

The aim of this study was to compare Campylobacter detection rates by microscopic examination to those based on culture in routine stool samples and to analyze the possible impact of performing Campylobacter culture as a routine method on the epidemiological data of campylobacteriosis in our clinical setting.

\section{Methods}

The study was conducted between January and April 2014 in the Clinical Laboratory of Clínica Alemana in Santiago, Chile. It utilized consecutive stool samples that were tested as part of routine care, which included culture methods for Salmonella, Shigella, Yersinia, and Vibrio, as well as direct stain for Campylobacter. Samples consisted of fresh stool, transported at ambient temperature and processed within $2 \mathrm{~h}$ after collection. For culture and enrichment, commercial selective media such as Mac Conkey, Salmonella-Shigella agar, CIN, TCBS, selenite broth, alkaline peptone water (all bioMérieux, l'Etoile, France) were used following standard recommendations $[2,15,16]$. Campylobacter staining was performed as recommended in Chile [13] and previously described [14, 17]. In short, smears of undiluted stool were prepared using a swab (155C, Copan, Brescia, Italy) and air dried. Then, slides were flooded for 1-2 min with a monthly prepared solution of equal parts of $1 \%$ sodium bicarbonate and commercial crystal violet solution (Color Gram 2 R1, bioMérieux). Slides were examined during routine workflow by a trained technician (50 high power oil immersion fields) for the presence of S-shaped or spiral rods. For quality control, all positive or doubtful slides were sent to the National Reference Laboratory. Each sample was inoculated with a swab on Campylobacter culture medium (Campylosel agar, bioMérieux), streaked into 4 quadrants with a sterile loop and incubated at $42{ }^{\circ} \mathrm{C}$ under microaerobic conditions (Anaerocult ${ }^{\circ} \mathrm{C}$, Merck, Darmstadt, Germany). Quality control for each batch of culture medium was performed using $C$. jejuni ATCC 33291 (growth at $48 \mathrm{~h}$ ) and E. coli ATCC 25922 (growth inhibition) as recommended [18]. Campylobacter plates were read after $48 \mathrm{~h}$ and suspicious colonies were further identified by matrix-assisted laser desorption/ionization time-of-flight (MALDI-TOF) technology using VITEK MS (bioMérieux). Following surveillance regulations, all enteropathogens were sent to the National Reference Laboratory for confirmation and susceptibility testing. Campylobacter strains were studied against ciprofloxacin, erythromycin and tetracycline by E-Test (bioMérieux) on blood Mueller-Hinton agar incubated for $24 \mathrm{~h}$ at $42{ }^{\circ} \mathrm{C}$ under microaerobic conditions. Results were interpreted according to CLSI guidelines [19].

The performance of Campylobacter staining technique was evaluated using culture as gold standard. To estimate the impact of culture methods on the detection of Campylobacter, isolation rates of all enteric bacterial pathogens within the study period were compared to those of the same time span of past years in our laboratory. These data were obtained from the laboratory databases. To compare isolation rates, $95 \%$ confidence intervals were calculated and the $\mathrm{Z}$ test for comparing two independent proportions was applied. $P$ values $<0.05$ were considered significant. For statistical analysis, Clinical Calculator 1 (http://vassarstats.net) was used.

\section{Results}

A total of 750 stool samples were examined. In 96 of them (12.8\%), bacterial pathogens were detected (Table 1). All enteric pathogens were identified to the species level with high identification scores (99.9\%) by Vitek MS.

Campylobacter culture was positive in 46 (6.1 \%) samples. The median age of the Campylobacter positive patients was 12 years (range: 1 to 89). The majority (41/46; $89.1 \%$ ) of strains was $C$. jejuni, the other $10.9 \%$ were $C$. coli. Staining technique identified three samples as Campylobacter positive, all of which contained C. jejuni. Thus, the Campylobacter detection rate using Hucker stain was $0.4 \%$ (Table 1 ) with a sensitivity of $6.5 \%$ (CI95 \%: $1.7-$ 18.9) and a specificity of $100 \%$ (CI95 \%: 99.3-100). Positive and negative predictive values for staining were $100 \%$ (CI95 \% 31.0-100) and 94.2\% (CI95 \% 92.3-95.8), respectively.

The culture-based Campylobacter rate within our study period (January to April 2014) was significantly higher than the staining-based rates of previous years (Fig. 1). A comparison of detection rates of all bacterial enteropathogens 
Table 1 Number and rate of bacterial pathogens identified in 750 routine stool samples

\begin{tabular}{llll}
\hline Pathogen & $\mathrm{n}$ & Isolation rate (\%) & $\mathrm{Cl} 95 \%$ \\
\hline Salmonella enterica & 49 & 6.5 & $4.9-8.6$ \\
Enteritidis & 33 & & \\
Montevideo & 7 & & \\
Heidelberg & 2 & & \\
Saintpaul & 2 & & \\
Sandiego & 1 & & \\
Othmarschen & 1 & & \\
Infantis & 1 & & $4.6-8.1$ \\
Derby & 1 & & \\
Not typed & 1 & & \\
Campylobacter (by culture) & 46 & 6.1 & $0.1-1.3$ \\
C. jejuni & 41 & & $0.01-0.9$ \\
C. coli & 5 & & $0.01-0.9$ \\
Campylobacter spp. (by staining) & 3 & 0.4 & $10.5-15.5$ \\
Yersinia enterocolitica & 1 & 0.1 & 12.8 \\
Vibrio parahaemolyticus & 1 & 0.1 & \\
Total & $96^{\mathrm{a}}$ & 12.8 & \\
\hline
\end{tabular}

${ }^{a}$ Including one sample with double infection, C. jejuni and $Y$. enterocolitica

identified in stool samples in our laboratory in the months of January to April of 2010 to 2014 demonstrated a clear change of the epidemiological relevance of Campylobacter after the introduction of culture methods in 2014 (Fig. 2). In the years 2010 to 2013, Salmonella was clearly the most frequently isolated enteropathogen, whereas the other bacterial causes were only rarely detected. In 2014, with the introduction of Campylobacter culture, Campylobacter became, together with Salmonella, the leading cause of bacterial enteritis in our patient collective.
National Reference Laboratory studied fourteen of the Campylobacter strains for antimicrobial susceptibility. Of those, 13 (92.8\%) were susceptible to erythromycin, whereas tetracycline- and ciprofloxacin-resistance was detected in three $(21.4 \%)$ and five $(35.7 \%)$ of the isolates, respectively.

\section{Discussion}

Campylobacter is a leading cause of gastrointestinal infections in industrialized and non-industrialized countries [1]. Its clinical and epidemiological importance is underlined by the ubiquitous nature of the pathogen, the possibility of severe post-infectious sequelae, and the increase of its burden during the last decade. Unfortunately, underreporting of campylobacteriosis is common and in some regions, the epidemiology of this infection is incompletely understood [5].

In most South American countries, the epidemiological role of Campylobacter as an enteric pathogen is unknown, since systematic studies are scant and national surveillance programs are mostly absent $[1,5]$. The main reason for this lack of data is that Campylobacter culture is expensive or not available and therefore, rarely included in the routine stool workup in microbiological laboratories [14]. In Chile, enteric Campylobacter infections were included in the national mandatory decree of active laboratory surveillance of enteropathogens in 1993 [7]. Since then, due to financial limitations, most Chilean laboratories adopted staining methods such as Hucker stain as the routine diagnostic tool for Campylobacter as suggested by local recommendations [13, 14]. The presented study highlights a potential problem of this approach since direct stool microscopy proved to have a very low and insufficient sensitivity compared to culture. In a previous study using the same technique in Chile, the sensitivity

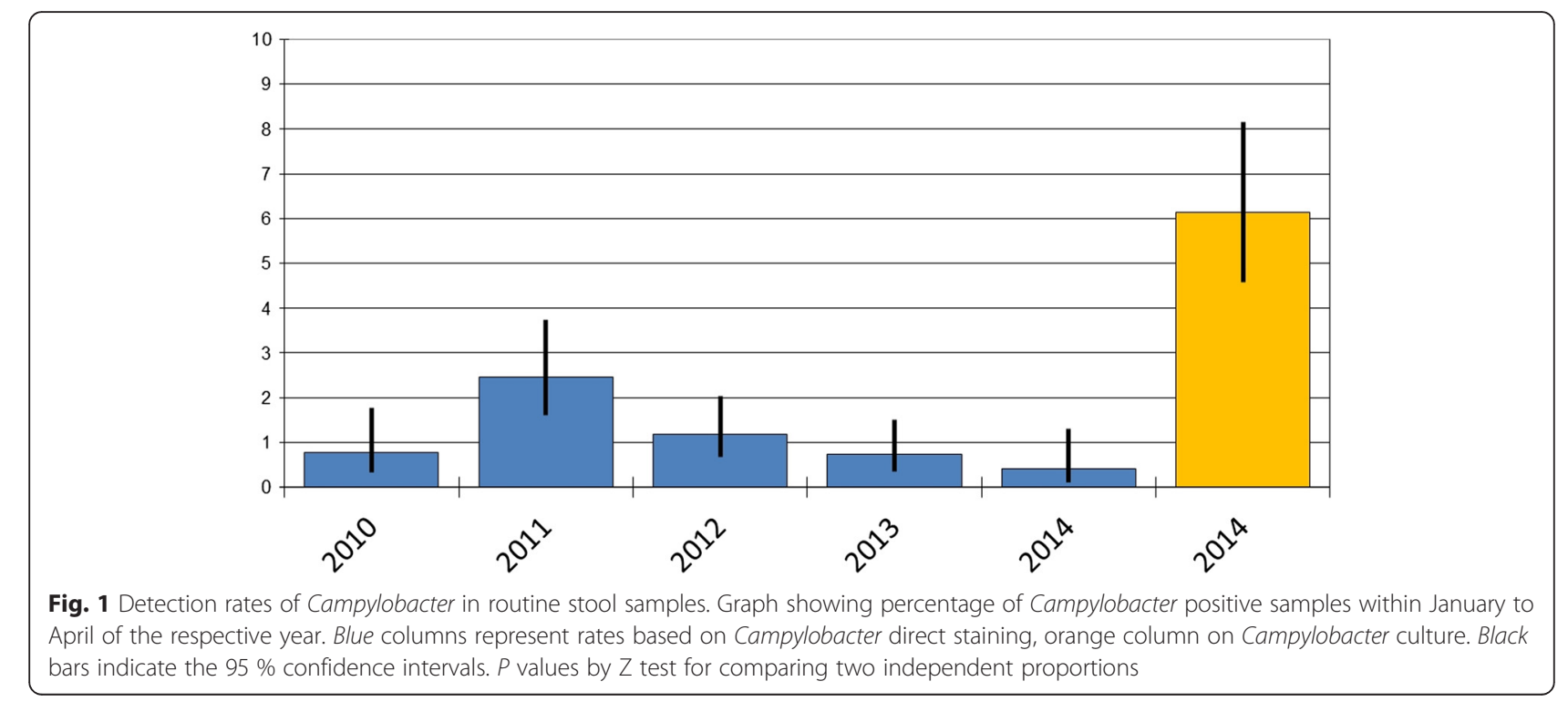




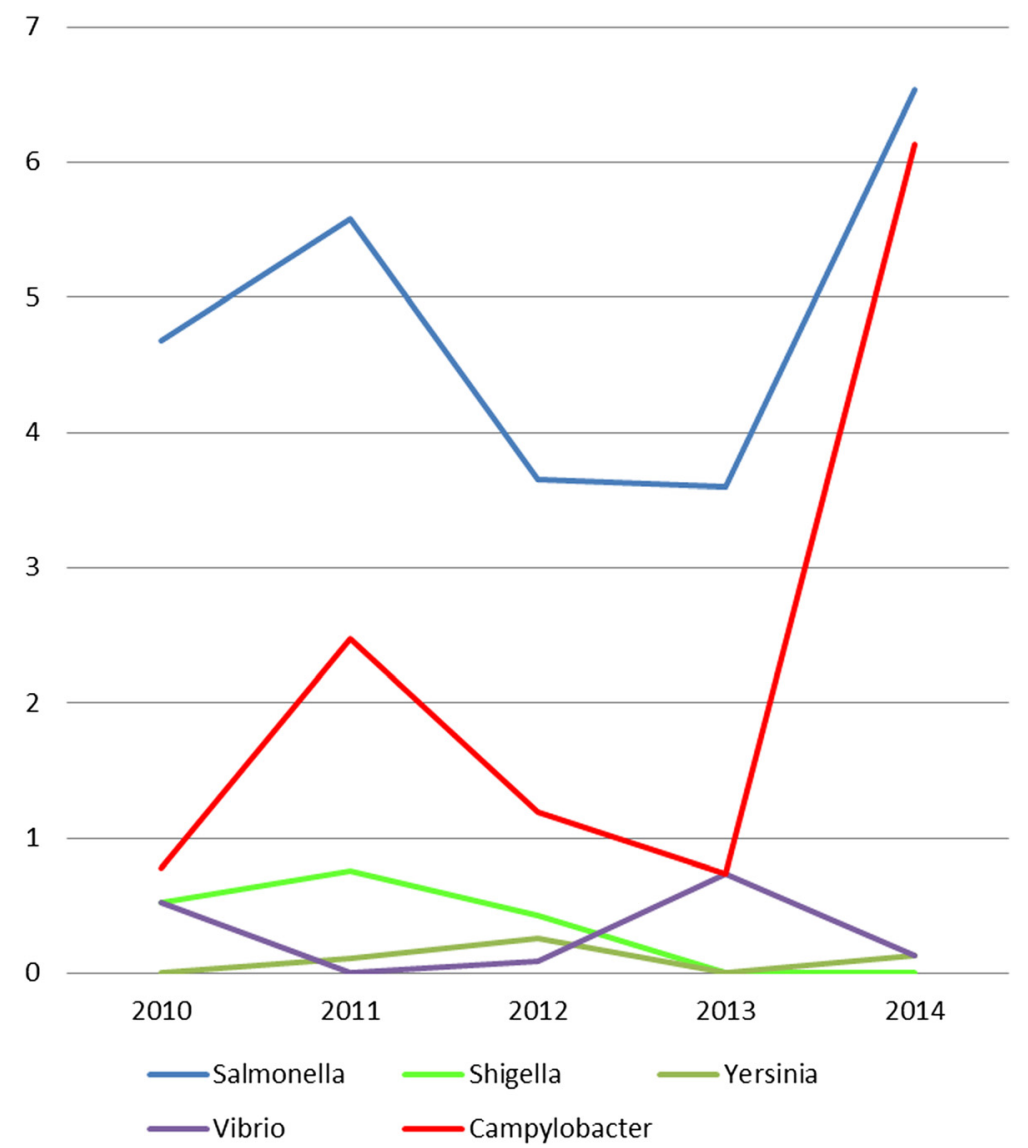

Fig. 2 Detection rates of enteric bacterial pathogens in routine stool samples. Graph showing percentage of enteropathogenic bacteria detected in stool samples within January to April of the respective year. All numbers are based on culture techniques except Campylobacter rates of the years 2010 to 2013, which are based on staining method

was higher, but the difference was not statistically significant (37.5\%, CI95 \% 16.3-64.1) [14]. Other studies using different stains such as Gram, reported much higher sensitivity values from 44 to $94 \%$ [20-24]. Reasons for the lower sensitivity in our study might be the use of a different staining technique, diagnostic settings (study versus routine conditions), study populations (children versus adults), and technical problems with Campylobacter culture, especially in older reports or in studies from developing countries. A main obstacle is that direct staining methods are highly operator dependent because they require significant expertise [2]. Therefore, objective evaluations of the performance are difficult and some experts discourage the routine use of these methods [15]. Another obstacle for a standardization of these techniques is the lack of protocols for quality control. External quality control programs offered from international institutions such as the College of American Pathologists (CAP) do not include direct Campylobacter staining. To overcome this limitation, microscopists should regularly be re-trained and tested. In accordance with other reports, the specificity of Campylobacter staining in our study was high [14,
20-24]. Positive results therefore, provide rapid and clinically relevant information. Still, direct staining can only serve as a supplemental test and should not replace Campylobacter culture.

Besides the costs, a drawback of Campylobacter culture is the prolonged incubation period. Still, the optimum time is controversial. While in North America, $72 \mathrm{~h}$ of incubation are recommended [2], British and German guidelines suggest shorter incubation periods of 40-48 h $[25,26]$. As a recent survey of more than 400 laboratories in the USA revealed, most participants also used $48 \mathrm{~h}$ for routine incubation for Campylobacter culture [27]. In our study we incubated cultures for $48 \mathrm{~h}$ as currently recommended by the Chilean consensus statement [13].

Culture-independent methods for Campylobacter diagnosis are commercially available in many countries in the industrialized world. Such tests include the detection of Campylobacter specific antigen and nucleic acids in stool samples. Until now, these tests are insufficiently evaluated to replace the traditional culture methods and do not serve for susceptibility testing and public health surveillance purposes [2]. The WHO encourages further research to 
validate the usefulness of these tests in low- and middleincome countries [1]. A recent study with samples from Tanzania, Bangladesh, and Peru showed higher Campylobacter prevalence rates than with culture, but no clear association with diarrheal disease [28]. In Chile, PCR detected higher prevalence of campylobacteriosis than culture, but most of the additional cases were caused by emerging Campylobacter species such as C. concisus [9]. To our opinion, the introduction of such new culture-independent techniques in countries with uncertain epidemiology such as Chile seems problematic at present.

With the inclusion of culture methods in routine stool examination during the study period, the Campylobacter detection rate increased by more than 10-fold compared to staining and was also significantly higher than in the same time period of the previous years (Fig. 1). Since a comparison of the staining-based rates did not show any evidence of an epidemiological change, we assume that the emergence of Campylobacter was truly related to the implementation of the new diagnostic procedure. A comparison of the detection rates of all bacterial enteropathogens during the years of 2010 to 2014 revealed that after the inclusion of culture, Campylobacter became a leading enteric pathogen with rates similar to those of Salmonella. This was a surprising finding, since in the official surveillance reports of recent years, Salmonella largely exceeds Campylobacter. In 2011 and 2012, for example, the respective numbers of cases were 3627 and 3076 for Salmonella, but only 170 and 136 for Campylobacter [7]. We believe that these extremely low rates of Campylobacter isolates might rather reflect on the insufficient diagnostic capacities to detect this pathogen than on the true epidemiological situation. This misjudgment and lack of reliable data leads to an underestimation of the importance of Campylobacter and further ignorance regarding its diagnosis - an epidemiological vicious circle. To our opinion, the implementation of Campylobacter culture as the routine diagnostic method in Chile is recommendable, although an exact analysis of the costeffectiveness of this method is pending.

Matrix-assisted laser desorption/ionization time-offlight (MALDI TOF) proved to be a rapid, convenient, and reliable diagnostic tool for Campylobacter species identification. If available, this technology might help to better understand the distribution of species other than C. jejuni. In our study, C. coli was less frequently isolated $(5 / 46 ; 10.9 \%)$ than previously reported in diarrhea cases in southern Chile (27.7 \%) [6].

Our data confirmed the high percentage of ciprofloxacin resistance of Campylobacter jejuni in Chile, which has previously been reported [29]. Surprisingly, one of the strains was also resistant to erythromycin, which had not been reported before in strains of human origin in Chile. These findings support the need for routine Campylobacter culture and for further surveillance of resistance.
The age distribution of our patients differed from older data in Chile, which reported that the majority of cases occurred in infants and preschool children [14], but was in accordance with a more recent study from southern Chile [9]. We observed a median age of 12 years without predominance of children less than 5 years. This might be explained by the high socioeconomic status of our patients attending a private hospital, since in North America and Europe campylobacteriosis in individuals older than ten years was associated with higher socioeconomic conditions [30].

Limitations of this study were that it covered only a limited period of time and samples derived only from a single clinical center. This might have aggravated the operator dependency of the staining method. As a study based on routine samples, the clinical data were limited. Furthermore, we did not include the study of viral or parasitic etiologies of enteritis.

\section{Conclusions}

Our study showed that with the inclusion of Campylobacter culture in the routine bacteriological stool workup, this pathogen became a leading cause of intestinal infection in our patient population. Direct Campylobacter staining methods, which have been promoted for resourcelimited settings, proved to be of low sensitivity and lead to a significant underestimation of the incidence of campylobacteriosis.

\section{Ethics statement}

The study was approved by the Comité de Ética de la Investigación ( $\mathrm{N}^{\circ}$ 2014-73), Centro de Bioética, Universidad del Desarrollo-Clínica Alemana, Santiago, Chile. Informed consent was waived since the study was performed within routine diagnostic procedures and did not involve intervention or interactions with patients.

\section{Consent to publish}

Not applicable.

\section{Availability of data and materials}

All the data supporting our findings is contained within the manuscript.

\section{Competing interests \\ The authors declare that they have no competing interests.}

\section{Authors' contributions}

CV and SM carried out the microbiology laboratory work and revised the manuscript. TH participated in the design of the study, performed the statistical analysis, and revised the manuscript. LP and TW designed and coordinated the study, and wrote the manuscript. All authors read and approved the final version of the manuscript.

\section{Acknowledgements}

We thank Roberto Flores and Oscar Duery for the information regarding the susceptibility testing performed in the Instituto de Salud Pública in Santiago. 
The data of this work were presented in part as a poster at the 54th Interscience Conference on Antimicrobial Agents and Chemotherapy (ICAAC), Washington DC, 5 to 9 September 2014.

\section{Funding}

No funding was obtained for this study.

Received: 26 August 2015 Accepted: 5 May 2016 Published online: 13 May 2016

\section{References}

1. WHO. The Global view of campylobacteriosis, report of an expert consultation. Utrecht, Netherlands, 2012. http://www.who.int/iris/bitstream/ 10665/80751/1/9789241564601_eng.pdf. Accessed 20 Aug 2015.

2. Fitzgerald C, Nachamkin I. Campylobacter and Arcobacter. In: Jorgensen JH, Pfaller MA, editors. Manual of clinical microbiology. Washington: ASM Press; 2015. p. 998-1012.

3. Ramonaite S, Kudirkiene E, Tamuleviciene E, Leviniene G, Malakauskas A, Gölz G, et al. Prevalence and genotypes of Campylobacter jejuni from urban environmental sources in comparison with clinical isolates from children. J Med Microbiol. 2014;63:1205-13.

4. Enberg J. Contributions to the epidemiology of Campylobacter infections. Dan Med Bull. 2006:53:361-89.

5. Kaakoush N, Castaño-Rodríguez N, Mitchell H, Man SM. Global epidemiology of Campylobacter infection. Clin Microbiol Rev. 2015:28:687-719.

6. Fernández H. Campylobacter and campylobacteriosis: a view from South America. Rev Peru Med Exp Salud Publica. 2011;28:121-7.

7. Instituto de Salud Pública de Chile. Vigilancia de laboratorio de Campylobacter sp. Chile, 2005 - 2013. In: Boletín Instituto de Salud Pública, Vol. 4, No. 1, Enero 2014. http://www.ispch.cl/sites/default/files/ Boletín\%20Campylobacter.pdf. Accessed 20 Aug 2015.

8. Crim SM, Griffin PM, Tauxe R, Marder EP, Gilliss D, Cronquist AB, et al. Centers for Disease Control and Prevention (CDC). Preliminary incidence and trends of infection with pathogens transmitted commonly through food - Foodborne Diseases Active Surveillance Network, 10 U.S. sites, 2006-2014. MMWR Morb Mortal Wkly Rep. 2015;64:495-9.

9. Collado L, Gutiérrez M, González M, Fernández H. Assessment of the prevalence and diversity of emergent campylobacteria in human stool samples using a combination of traditional and molecular methods. Diagn Microbiol Infect Dis. 2013;75:434-6.

10. Fernández H, Kahler K, Salazar R, Ríos M. Prevalence of thermotolerant species of Campylobacter and their biotypes in children and domestic birds and dogs in southern Chile. Rev Inst Med Trop Sao Paulo. 1994;36:433-6.

11. Figueroa G, Galeno H, Troncoso M, Toledo S, Soto V. Prospective study of Campylobacter jejuni infection in Chilean infants evaluated by culture and serology. J Clin Microbiol. 1989;27:1040-4.

12. Platts-Mills J, Kosek M. Update on the burden of Campylobacter in developing countries. Curr Opin Infect Dis. 2014;27:444-50.

13. Comité de Microbiología Clínica (Sociedad Chilena de Infectología), Laboratorio de Referencia de Bacteriología (Instituto de Salud Pública), Instituto de Ciencias Biomédicas (Facultad de Medicina, Universidad de Chile). Acute diarrheal syndrome: recommendations for the microbiological diagnosis. Rev Chilena Infectol. 2002;19:1-13.

14. Chanqueo L, García P, León E, Blu F. Evaluation of Hucker stain as Campylobacter sp screening detection during an acute diarrhea disease. Rev Chilena Infectol. 2005;22:242-6.

15. Humphries $R$, Linscott $A$. Laboratory diagnosis of bacterial gastroenteritis. Clin Microbiol Rev. 2015:28:3-31.

16. On S. Isolation, identification and subtyping of Campylobacter: where to from here? J Microbiol Methods. 2013:95:3-7.

17. Valenzuela ME, D'Ottone K. Diagnóstico presuntivo rápido de enteritis asociada a Campylobacter. Rev Chil Infect. 1984;2:132-4.

18. NCCLS. Quality control for commercially prepared microbiological culture media; approved standard - third edition, NCCLS document M22-A3. NCCLS, 940 West Valley Road, Suite 1400, Wayne, Pennsylvania 19087-1898 USA 2004. ISBN 1-56238-536-4.

19. Clinical and Laboratory Standards Institute (CLSI). Methods for antimicrobial dilution and disk susceptibility testing of infrequently isolated or fastidious bacteria, CLSI guideline M45. 3rd ed. Wayne: Clinical and Laboratory Standards Institute; 2015. ISBN 1-56238-917-3.
20. Sazie ES, Titus AE. Rapid diagnosis of campylobacter enteritis. Ann Intern Med. 1982;96:62-3.

21. Ho DD, Ault MJ, Ault MA, Murata GH. Campylobacter enteritis: early diagnosis with Gram's stain. Arch Intern Med. 1982;142:1858-60.

22. Park CH, Hixon DL, Polhemus AS, Ferguson CB, Hall SL, Risheim CC, et al. A rapid diagnosis of Campylobacter enteritis by direct smear examination. Am J Clin Pathol. 1983:80:388-90.

23. Wang $\mathrm{H}$, Murdoch DR. Detection of Campylobacter species in faecal samples by direct Gram stain microscopy. Pathology. 2004;36:343-4.

24. Mushi MF, Paterno L, Tappe D, Deogratius AP, Seni J, Moremi N, et al. Evaluation of detection methods for Campylobacter infections among under-fives in Mwanza City, Tanzania. Pan Afr Med J. 2014;19:392.

25. Public Health England (2015). Identification of Campylobacter species. UK Standards for Microbiology Investigations. ID23 Issue 3. https://www.gov.uk/ uk-standards-for-microbiology-investigations-smi-quality-and-consistency-inclinical-laboratories. Accessed Dec. 29, 2015.

26. Kist M. Campylobacter und Arcobacter spp. In: Neumeister B, Geiss HK, Braun RW, Kimmig P, editors. Mikrobiologische Diagnostik. Stuttgart, New York: Georg Thieme Verlag; 2009. p. 565-73.

27. Hurd S, Patrick M, Hatch J, Clogher P, Wymore K, Cronquist AB, et al. Clinical laboratory practices for the isolation and identification of Campylobacter in Foodborne Diseases Active Surveillance Network (FoodNet) sites: baseline information for understanding changes in surveillance data. Clin Infect Dis. 2012;54 Suppl 5:S440-5.

28. Platts-Mills JA, Liu J, Gratz J, Mduma E, Amour C, Swai N, et al. Detection of Campylobacter in stool and determination of significance by culture, enzyme immunoassay, and PCR in developing countries. J Clin Microbiol. 2014:52:1074-80.

29. García P, Valenzuela N, Rodríguez M, León E, Fernández H. Susceptibilidad antimicrobiana de Campylobacter jejuni aislado de coprocultivos en Santiago de Chile. Rev Chilena Infectol. 2009;26:511-4.

30. Bemis K, Marcus R, Hadler J. Socioeconomic status and campylobacteriosis, Connecticut, USA, 1999-2009. Emerg Infect Dis. 2014:20:1240-1.

\section{Submit your next manuscript to BioMed Central and we will help you at every step:}

- We accept pre-submission inquiries

- Our selector tool helps you to find the most relevant journal

- We provide round the clock customer support

- Convenient online submission

- Thorough peer review

- Inclusion in PubMed and all major indexing services

- Maximum visibility for your research

Submit your manuscript at www.biomedcentral.com/submit
) Biomed Central 